\title{
Gas-Phase Reactions of Divalent Ni Complex Ions with Acetonitrile: Chelate Ring Size, Inductive, and Steric Effects
}

\author{
Marianny Y. Combariza and Richard W. Vachet \\ Department of Chemistry, University of Massachusetts, Amherst, Massachusetts, USA
}

\begin{abstract}
$\mathrm{Ni}(\mathrm{II})$ complexes of a series of pentadentate polyamine ligands have been reacted with $\mathrm{CH}_{3} \mathrm{CN}$ in the gas phase using a modified quadrupole ion trap mass spectrometer. The ligands have structural features such that upon complexation, chelate ring size, sterics, and inductive effects can be evaluated in the gas phase. Rate and equilibrium constants for $\mathrm{CH}_{3} \mathrm{CN}$ addition to the metal complexes show that there is a general decrease in the gas-phase reactivity as the chelate ring size is increased. Density functional theory calculations at the B3LYP/LANL2DZ level of theory have been used to obtain minimum energy structures and Mulliken charges for the complexes. The decreased reactivity observed as the chelate ring size is increased correlates with a decrease in the atomic charge on the metal. A larger chelate ring size enhances ligand flexibility and improves the overlap of the ligand's donor atoms with the metal center. Adding methyl groups adjacent to or on the nitrogen donor groups of a ligand also decreases the rate and equilibrium constants for the reactions of a given complex with $\mathrm{CH}_{3} \mathrm{CN}$. Analysis of Mulliken charges for these complexes indicates that both inductive and steric effects are responsible for lower complex reactivity. These results suggest that while the gas-phase reactivity of a metal complex with $\mathrm{CH}_{3} \mathrm{CN}$ is very dependent on the functional groups directly bound to the metal, in some cases steric effects can conceal the correlation between reactivity and coordination structure. (J Am Soc Mass Spectrom 2004, 15, 1128-1135) (C 2004 American Society for Mass Spectrometry
\end{abstract}

$\mathrm{T}$ The chemistry of transition metal ions is quite diverse mainly because they can be tuned by the type, number, and orientation of the ligands coordinated to them. Knowledge of this coordination sphere provides insight into metal complex reactivity, and a variety of techniques including $\mathrm{X}$-ray spectroscopies, NMR, and electron paramagnetic resonance (EPR) have been used to gather coordination structure information. If metal complexes are present at low concentrations or in complicated mixtures, though, these techniques can have difficulty providing coordination structure. Consequently, we have begun to investigate the advantage of using mass spectrometry (MS) to gather coordination structure information.

For several reasons we have chosen not to rely on the typical dissociation methods (e.g., collision-induced dissociation [CID]) for acquiring metal complex structural information by MS. One reason is that, while the dissociation patterns and dynamics of metal complexes have not been exhaustively studied, most results indicate that rearrangement reactions involving the loss of small neutrals from large ligands or the loss of intact

Published online June 19, 2004

Address reprint requests to Dr. R. W. Vachet, Department of Chemistry, University of Massachusetts, 701 Lederle GRT, 710 North Pleasant Street, Amherst, MA 01003, USA. E-mail: rwvachet@chem.umass.edu ligands from multi-ligated metal ions are typical [1-11]. Unfortunately, neither provides much insight into the coordination sphere, as they do not necessarily reveal whether the groups lost were coordinated to the metal or not. Furthermore, during collisional activation, even at low energies, scrambling of the metal's coordination sphere can occur because of relatively weak metalligand interactions. As a result rearrangements can occur, and the structure from which a metal complex dissociates may differ from its initial structure. Given these drawbacks to the use of CID for coordination structure analysis, we have begun to investigate the potential of using low energy ion-molecule reactions that are gentler.

We have shown that ion-molecule (I-M) reactions can be sensitive enough to distinguish complexes with different coordination numbers [12-17], coordinating functional groups [18], and coordination geometries $[19,20]$. Using I-M reactions to provide coordination structure relies on reacting metal complexes of interest with reagent molecules such as $\mathrm{CH}_{3} \mathrm{CN}$ and $\mathrm{NH}_{3}$. Addition of these ligands provides an easily measured mass increase, and the extent of the reaction provides insight into the structure. Because this approach relies on complexation of a reagent ligand, steric factors that might affect these reactions need to be evaluated. Consequently, this work describes the gas-phase reactions 


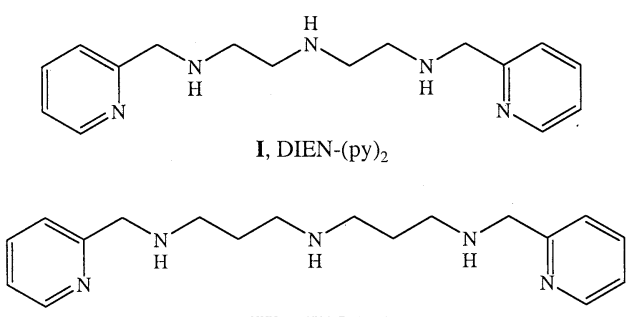

III, DIPN-(py) 2
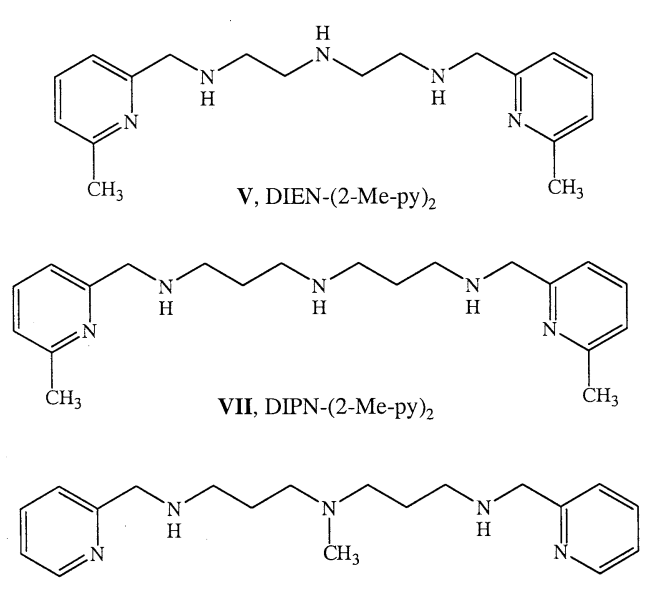

IX, Me-DIPN-(py)

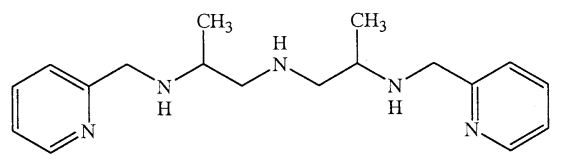

II, DI-iPN-(py) 2
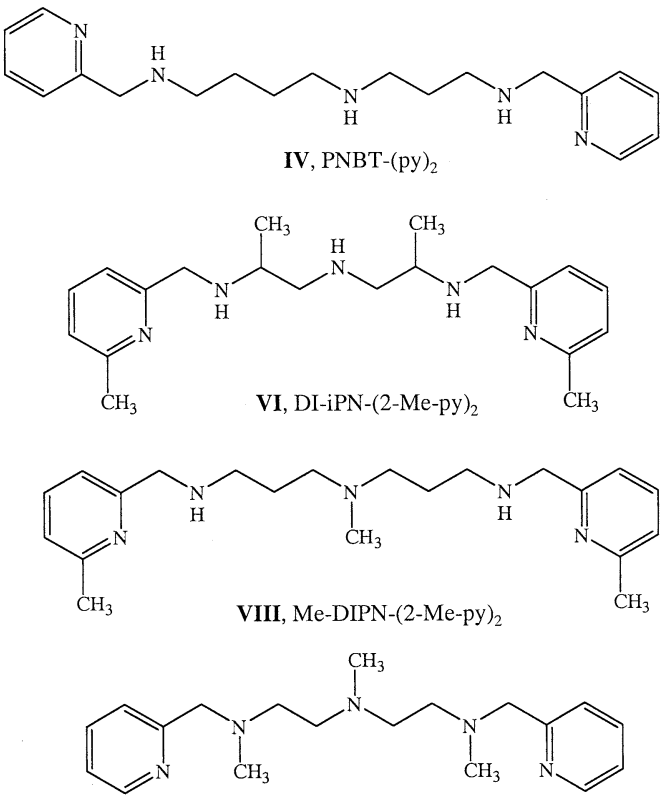

X, Tri-Me-DIEN-(py) 2

Figure 1. The ligands used in this study.

of a series of metal complexes with pentadentate polyamine ligands, which differ only in the placement of various methyl and methylene groups. These ligands and their resulting metal complexes allow us to investigate the interrelated effects of sterics, induction, and chelate ring size on complex reactivity.

\section{Experimental}

\section{Ligand and Metal Complex Synthesis}

The ligands used in this study (Figure 1) were synthesized by attaching pyridine and methylpyridine groups to the ends of a series of triamines. Ligands I, II, III, and IX were synthesized by mixing bis(2-aminoethyl)amine (DIEN), bis(2-aminopropyl)amine (DI-iPN), N-(3-aminopropyl)-1,3-propanediamine (DIPN), or 3,3'-diamino$\mathrm{N}$-methyldipropylamine (Me-DIPN) with 2-pyridinecarboxaldehyde in a 1:2 ratio (triamine:aldehyde). Ligands V, VI, VII, and VIII were prepared by mixing the same triamines with 6-methylpyridine-2-carboxaldehyde in a 1:2 ratio (triamine:aldehyde). $\mathrm{N}$-(3-aminopropyl)-1,4-diaminobutane (PNBT) and 1,4,7-trimethyldiethylenetriamine (Tri-Me-DIEN) were mixed with 2-pyridinecarboxaldehyde in a 1:2 ratio to form Ligands IV and X. In every case the resulting Schiff bases were then reduced for $24 \mathrm{~h}$ in a Parr shaking hydrogenator under a 60 psi atmosphere of $\mathrm{H}_{2}$ over $\mathrm{Pd}(10 \% \mathrm{Pd}$ on carbon). All chemicals were obtained from Sigma-Al- drich (St. Louis, $\mathrm{MO}$ ) and were used without further purification. $\mathrm{H}_{2}(99.99 \%)$ was obtained from Merriam Graves (Springfield, MA). Additional details for the syntheses of these ligands can be found in previous publications [18, 21].

Metal complexes were prepared at a concentration of $100 \mu \mathrm{M}$ by mixing $\mathrm{NiCl}_{2}$ solutions with an equal amount of the ligand of interest in $100 \%$ methanol or water:methanol (1:1). Electrospray ionization, with a needle voltage of about $4 \mathrm{kV}$ and a flow rate of 1.0-2.0 $\mu \mathrm{L} / \mathrm{min}$, was used to generate the complex ions $\left(\mathrm{ML}^{2+}\right)$. Normally, a capillary temperature of $150{ }^{\circ} \mathrm{C}$ and a capillary exit offset voltage of $20-30 \mathrm{~V}$ were used.

\section{Ion-Molecule Reactions}

I-M reaction experiments were performed in a modified Bruker ESQUIRE-LC quadrupole ion trap mass spectrometer. Details of the modifications made to this instrument are provided elsewhere [18]. $\mathrm{CH}_{3} \mathrm{CN}$ was used as the reagent gas at a pressure of $3.2 \pm 0.3 \times 10^{-7}$ torr, and the helium buffer gas was maintained at a pressure of $1.0 \pm 0.3 \times 10^{-4}$ torr. The vacuum system temperature was monitored with a thermocouple and maintained at $300 \pm 2 \mathrm{~K}$ using a heating blanket. Doubly-charged complex ions were isolated and reacted with $\mathrm{CH}_{3} \mathrm{CN}$ in the gas phase for different periods of time. The ESQUIRE-LC software was used to control 
ion selection and reaction time. Kinetic data were obtained by monitoring the change in the abundances of product and parent ions for up to $4000 \mathrm{~ms}$. Rate constants for the I-M reactions were obtained by fitting the experimental kinetic data to a series of differential equations using the program KinFit [22]. The ratio of the forward and back rate constants provided equilibrium constants for the reactions. In cases where the fitting process failed to provide meaningful values for the back rate constants, equilibrium constants were calculated as the ratio of the product and parent ion abundances at equilibrium.

\section{Electronic Structure Calculations}

Density functional theory (DFT) calculations were carried out with the Gaussian suite of computational chemistry programs [23]. We calculated Ni(II) complexes of Ligands I-X and performed geometry optimizations at the B3LYP level [24-26] with the LANL2DZ basis set [27-30]. The use of an all-electron basis set was computationally expensive for the complexes in this study because of the size of the complexes and the presence of a transition metal center. The LANL2DZ basis set uses a combination of effective core potentials (ECP's), which substitute the core electrons in heavy atoms, and Gaussian orbital valence basis sets [27-30].

A search of the Cambridge Crystallographic Database retrieved structures for the $\mathrm{Ni}$ (II) complexes of DIEN-(py) $)_{2}$ and DIPN-(py) $)_{2}$, which were used as the starting points for the DFT calculations. Because no crystal data was found for any of the other complexes examined, the geometry optimized structures of $\mathrm{Ni}$ $\left(\mathrm{DIEN}-(\mathrm{py})_{2}\right)^{2+}$ and $\mathrm{Ni}\left(\mathrm{DIPN}-(\mathrm{py})_{2}\right)^{2+}$ were used as templates to construct their methyl analogs (complexes of Ligands I-III and V-X in Figure 1). Ni(DIPN-(py $\left.)_{2}\right)^{2+}$ was used to obtain a starting structure for the Ni(PNBT$\left.(\mathrm{py})_{2}\right)^{2+}$ complex by introducing the appropriate methylene groups. After adding the necessary methylene or methyl units to obtain the $\mathrm{Ni}$ (II) complexes of all the ligands in Figure 1, optimizations at the B3LYP/ LANL2DZ level of theory were performed.

The DFT calculations were used to obtain metal complex geometries and electronic distributions, which were then used to provide insight into the reactivity patterns observed in the gas phase. Given that electrons are not localized entities and atomic charges are not an observable property of a molecule, there is no precise way to describe these atomic charges. Nonetheless, many definitions have appeared in the literature, but perhaps the most common approach for calculating atomic charges is the Mulliken population analysis. Mulliken charges are derived from a simple partition of the electrons among the atomic orbital basis functions [31-34]. Mulliken charges reflect the polarities of atoms and functional groups, and they correlate well with an atom's electronegativity [31-35]. Mulliken charges for $\mathrm{Ni}$ were taken from the minimum energy structures of

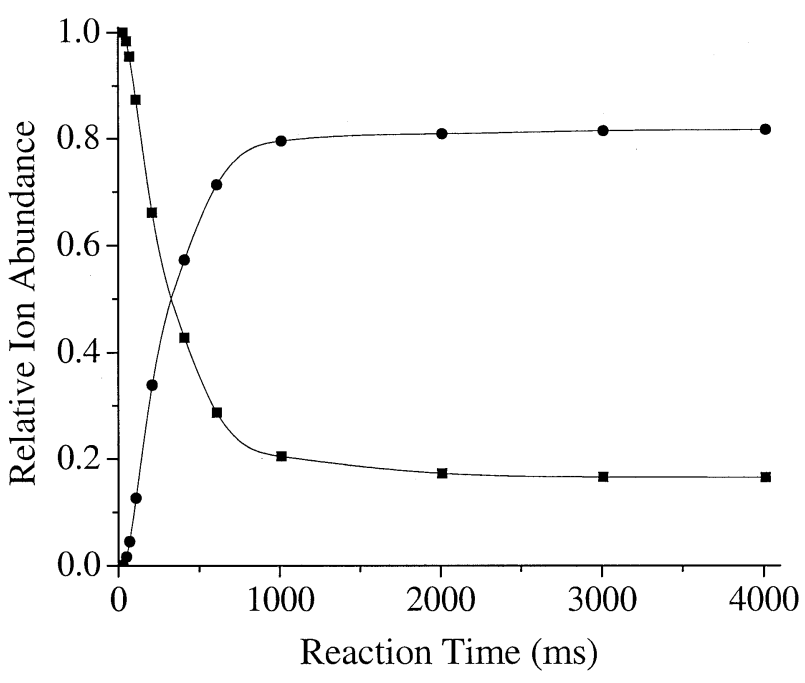

Figure 2. Typical experimental kinetic plot for the reaction of $\mathrm{Ni}\left(\mathrm{DIPN}-(\mathrm{py})_{2}\right)^{2+}$ with $\mathrm{CH}_{3} \mathrm{CN}$. The ion abundance of the parent ion, $\mathrm{Ni}$ (DIPN- $\left.(\mathrm{py})_{2}\right)^{2+}$, is indicated by a filled square, and the product ion, $\mathrm{Ni}\left(\mathrm{DIPN}-(\mathrm{py})_{2}\right)\left(\mathrm{CH}_{3} \mathrm{CN}\right)^{2+}$, is indicated by a filled circle.

the complexes found by performing the geometry optimizations.

Ligand strain energies for several ligands were also determined. The procedure to calculate ligand strain energy first involved obtaining a minimum energy structure for the metal complex at the B3LYP/ LANL2DZ level of theory. Then, the metal center was removed from the structure and a single-point energy calculation was performed on the "strained" ligand structure at the B3LYP/6-31G(d,p) level of theory. Finally, the "strained" ligand structure without the metal was allowed to "relax" by performing a geometry optimization at the B3LYP/6-31G(d,p) level of theory. With this procedure minimum energy values for the final structures were obtained, and the strain energy was calculated as the difference between the "strained" and "relaxed" ligand structures.

\section{Results and Discussion}

Previous results have shown that five-coordinate complexes add just a single reagent gas molecule to fill the metal's coordination sphere $[12,18]$. Ligands I-X have five potential binding sites, and their resulting complexes upon coordination to $\mathrm{Ni}$ (II) should add one $\mathrm{CH}_{3} \mathrm{CN}$ molecule in the gas phase. By following the evolution of parent and product ion abundances as a function of reaction time, kinetic plots for the reactions can be obtained. Figure 2 displays a typical experimental plot representing the normalized parent and product ion abundances versus reaction time for the reaction of $\mathrm{Ni}\left(\mathrm{DIPN}-(\mathrm{py})_{2}\right)^{2+}$ with $\mathrm{CH}_{3} \mathrm{CN}$. Examination of each kinetic plot shows that the reactions of all the $\mathrm{Ni}$ (II) complexes of Ligands I-X achieve an equilibrium after about $2000 \mathrm{~ms}$, and addition of a single $\mathrm{CH}_{3} \mathrm{CN}$ molecule is observed in each case. Fitting of the kinetic data 
Table 1. Rate and equilibrium constants for the reaction of various $\mathrm{Ni}(\mathrm{II})$ complexes with $\mathrm{CH}_{3} \mathrm{CN}$

\begin{tabular}{|c|c|c|c|c|}
\hline & Ligand & $\begin{array}{c}\text { Rate constant }{ }^{\mathrm{a}} \\
\left(\mathrm{x} 10^{-11} \mathrm{~cm}^{3} / \text { molecule-s }^{-}\right)\end{array}$ & $\begin{array}{l}\text { Equilibrium constant } \\
\qquad\left(\times 10^{9} \text { atm }^{-1}\right)\end{array}$ & Mulliken charge \\
\hline I & DIEN-(py) 2 & $40 \pm 10$ & $330 \pm 150$ & 0.52 \\
\hline II & DI-iPN-(py) ${ }_{2}$ & $40 \pm 10$ & $130 \pm 30$ & 0.49 \\
\hline III & DIPN-(py) 2 & $19 \pm 1$ & $9 \pm 2$ & 0.46 \\
\hline IV & PNBT-(py $)_{2}$ & $7.2 \pm 0.1$ & $1.2 \pm 0.1$ & 0.38 \\
\hline $\mathrm{V}$ & DIEN-(2-Me-py) 2 & $13.5 \pm 0.4$ & $2.3 \pm 0.7$ & 0.45 \\
\hline VI & DI-iPN-(2-Me-py)2 & $4.0 \pm 0.1$ & $0.23 \pm 0.03$ & 0.44 \\
\hline VII & DIPN-(2-Me-py) 2 & $2.2 \pm 0.2$ & $0.21 \pm 0.03$ & 0.39 \\
\hline VIII & Me-DIPN-(2-Me-py) ${ }_{2}$ & $3.4 \pm 0.1$ & $0.23 \pm 0.2$ & 0.39 \\
\hline $\mathrm{IX}$ & Me-DIPN-(py $)_{2}$ & $9 \pm 1$ & $4 \pm 2$ & 0.45 \\
\hline$X$ & Tri-Me-DIEN-(py $)_{2}$ & $1.4 \pm 0.1$ & $0.14 \pm 0.03$ & 0.45 \\
\hline
\end{tabular}

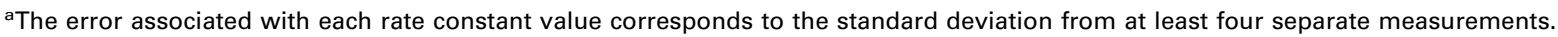

to eq 1 allows us to obtain rate and equilibrium constants for these reactions (see Table 1).

$$
\mathrm{ML}^{2+}+\mathrm{CH}_{3} \mathrm{CN} \underset{\mathrm{k}_{2}}{\stackrel{\mathrm{k}_{1}}{\longrightarrow}}\left(\mathrm{ML}+\mathrm{CH}_{3} \mathrm{CN}\right)^{2+}
$$

Table 1 clearly shows differences in reactivity for each complex. Because these are association reactions, the reaction rate constants and the equilibrium constants are both dependent upon the binding strength of the $\mathrm{CH}_{3} \mathrm{CN}$ to the metal complex. As we have shown in our previous work $[18,19,36]$, the magnitudes of both rate and equilibrium constants should generally follow the same trend as the nature, and thus the reactivity, of the metal complex changes. For simplicity, in the following discussion the equilibrium constant values will be used as the figures of merit to distinguish the reactivity of different complexes.

Table 1 clearly shows some remarkable changes in reactivity observed as the ligands are varied. Previously, we established that the ability of a ligand's donor groups to donate electron density dramatically affects the reactivity of the complex, and the extent of reagent ligand addition can be correlated with the coordination sphere of the complex $[18,36]$. In this work the ligand donor groups are only minimally changed. In all cases the donor groups are either secondary or tertiary amines. The most significant changes to the ligand structures correspond to the addition of methylene and/or methyl groups to the ligand framework. It is well known that chelate ring size, sterics, and inductive effects are key features that control metal-ion selectivity in both open-chain and macrocyclic ligands [37-39]. With Ligands I-X we have introduced structural features that allow the effect of such factors on the gasphase reactivity of the metal complexes to be evaluated. The complexes of Ligands I-IV allow the effects of chelate ring size to be notionally evaluated, although inductive and steric effects will also have a bearing on the reactivity of these complexes to some degree. The remaining ligands more directly allow steric and inductive effects to be examined.

\section{Chelate Ring Size Effects}

The results in Table 1 show that a general reduction in the rate and equilibrium constants is observed as the chelate ring size increases from five to seven. This observation is illustrated by the $\mathrm{Ni}$ (II) complexes of Ligands I, III, and IV. For many metal-ligand complexes in solution, five-membered chelate rings lead to greater complex stability than the presence of smaller or larger chelate ring sizes $[39,40]$. For metal-ligand complexes of high denticity (e.g., tetradentate ligands), however, it has been observed that a change in the chelate ring size from five to six actually stabilizes the complexes of small transition metal ions (e.g., $\mathrm{Ni}^{2+}, \mathrm{Cu}^{2+}, \mathrm{Zn}^{2+}$ ) but not larger metal ions (e.g., $\mathrm{Pb}^{2+}$ ) [41-46]. A reduction in the equilibrium constants is observed for the $\mathrm{Ni}(\mathrm{II})$ complexes of ligands I and III (Table 1) as the chelate ring size increases from five to six. This reduction correlates well with the expected stability constant increase in solution for ligands with similar denticity to the ligands in our study [41-43]. We have observed for numerous metal complex ions with the same denticity that the extent of a complex's gas-phase reactivity with a reagent is roughly related to the formation constant of that complex in solution [47]. In other words, more stable metal-ligand complexes have lower equilibrium constants for their gas-phase reactions with reagents like $\mathrm{CH}_{3} \mathrm{CN}$.

This correlation between the equilibrium constant in the gas phase reaction and the stability constant in solution has some limits, though, as evidenced by further reduction in the equilibrium constants when the chelate ring size is increased from six (Ligand III) to seven (Ligand IV). Solution-phase reports indicate that when the chelate ring size is increased from six to seven a substantial decrease in stability constants is observed, which is independent of the metal ion size [41-44]. The decrease in the gas-phase equilibrium constants for the reaction of $\mathrm{CH}_{3} \mathrm{CN}$ with the $\mathrm{Ni}$ (II) complex of Ligand IV could be caused by at least two factors. First, increasing the number of methylene groups adjacent to the nitrogen in the ligand may sterically hinder the addition of $\mathrm{CH}_{3} \mathrm{CN}$. This factor could also then be the cause of the decreased reactivity of the complexes of Ligand III 
when compared with the complexes of Ligand I. Second, the nitrogens along the backbone of Ligand IV may donate more electron density to the metal ion either because of more efficient overlap with the metal due to better dipole orientation or increased $\sigma$-donating capacity due to additional adjacent methylene groups.

To test these two possibilities, two sets of experiments were performed. First, the $\mathrm{Ni}(\mathrm{II})$ complex of Ligand II was reacted with $\mathrm{CH}_{3} \mathrm{CN}$. Complexes of this ligand maintain five-membered chelate rings, while at the same time introducing a methyl group adjacent to the nitrogen donor atoms. A comparison of the reactivity of the Ni(II) complex of Ligand II with the complex of Ligand I indicates that this modification has less of an effect on reactivity than a change in chelate ring size. This result suggests that the addition of extra methyl groups adjacent to the nitrogen donors does not likely decrease the reactivity of the complexes of Ligand III, and probably Ligand IV, for steric reasons. The second set of experiments involved using DFT to calculate the atomic charge on the metal center in each of the complexes of Ligands I-IV, and the resulting Mulliken charges are listed in Table 1. For the complexes of Ligands I-IV, the charges on Ni correlate directly with the experimentally determined equilibrium constants for the reactions of these complexes with $\mathrm{CH}_{3} \mathrm{CN}$. The trend indicates that as the charge on the metal decreases the reactivity of the complex decreases. This result is not surprising, and it agrees with our previous report $[18]$ and the work of others $[48,49]$ in which the ability of the ligand donor groups to donate electron density to the metal center was observed to directly affect complex reactivity. Essentially, greater metal-donor overlap, which leads to stronger metal-ligand bonds, decreases the charge on the metal and weakens the metal- $\mathrm{CH}_{3} \mathrm{CN}$ interaction, which leads to lower equilibrium constants. In the present case, as the chelate ring size increases the charge on the central metal ion decreases, and the reactivity of the complex with $\mathrm{CH}_{3} \mathrm{CN}$ decreases. This effect is likely caused by better overlap of the donor atoms with the metal center as a consequence of the increased flexibility of the ligand. Presumably the nitrogen donor atoms can more easily orient their dipoles toward the metal center. Ligand flexibility and optimal dipole orientation have also been invoked to explain the gas-phase dissociation behavior of metal complexes [21, 50-52]. The increased number of adjacent methylene groups might also increase the $\sigma$-donating capacity of the nitrogen donors in Ligands III and IV, leading to a decreased charge on $\mathrm{Ni}$; however, the minor changes in the Mulliken charge on $\mathrm{Ni}\left(\mathrm{DI}-\mathrm{iPN}-(\mathrm{py})_{2}\right)^{2+}$ and the reactivity of the $\mathrm{Ni}$ (II) complex of Ligand II indicate that the increase in $\sigma$-donating capacity is only minimal.

The decreased gas-phase reactivity upon going from Ligand I to III to IV might have implications for understanding the effect of chelate ring sizes in solution. Both the gas-phase equilibrium data and the DFT calculations suggest that there is an enthalpic benefit in going to larger chelate ring sizes. Of course, solution stability constants are dependent upon both the enthalpy and entropy of the metal-complex formation reaction. The higher solution stability constants of some metals (i.e., small metals like Ni(II)) with ligands of high denticity (i.e., tetradentate and higher) having six-membered chelate rings might then be understood by the well known trade-off between enthalpy and entropy. Even though favored more for enthalpic reasons, sevenmembered chelate rings are relatively unfavored for entropic reasons. In contrast, five-membered chelate rings are favored entropically, but they are more unfavored enthalpically. For Ni(II) six-membered chelate rings evidently provide the right balance of enthalpic and entropic contributions in solution. This same explanation was suggested after the initial observation that an increase in chelate ring size leads to a reduction in complex stability for relatively small transition metal ions [53]. After several studies, however, the reduction in complex stability as the chelate ring size increases is now usually explained in terms of the unfavorable enthalpy associated with steric strain and the difficulty of orienting donor dipoles toward the metal as the chelate ring size increases [39]. Calculations of ligand strain using the approach described in the Experimental section give values of 157,158 , and $172 \mathrm{~kJ} / \mathrm{mol}$ for the complexes of Ligands I, III, and IV, respectively, which is somewhat consistent with this explanation. Our experimental data and Mulliken-charge calculations, however, suggest that even if ligand strain is slightly increased with larger chelate ring sizes, the donor atoms can still orient themselves effectively to provide sufficient electron density to the metal.

While our gas-phase data and DFT calculations suggest a possible flaw in the ring strain explanation for the trend in solution stability constants as a function of chelate ring size, more experimental work is necessary to clarify this issue. Because our gas-phase reactions appear to measure the inherent enthalpic contribution from the ligand, we speculate that the trend in solution stability constants is a combination of both ligand solvation effects, which might prevent optimal orientation of the ligand donor groups toward the metal in solution, and entropic considerations. In future experiments we will carry out gas-phase reactions and DFT calculations on a wider range of chelate complexes with a particular emphasis on those complexes that in solution have been well studied with respect to chelate ring size [39].

\section{Steric and Inductive Effects}

As noted above, the less extensive reactivity of the $\mathrm{Ni}$ (II) complexes as methylene groups are introduced onto the ligand backbone (i.e., Ligands I, III, and IV) is likely a result of better metal-donor overlap rather than increased sterics. Placement of methyl groups at other positions in the ligand (i.e., Ligands $\mathrm{V}-\mathrm{X}$ ) also decreases complex reactivity (see Table 1); however, the decreased reactivity appears to be the result of increased 
sterics in these cases instead of just simple inductive effects. A comparison of the reactivity of the complexes of Ligands I, II, and III with analogs V, VI, and VII, in which methyl groups have been introduced into the pyridine rings, indicates a significant decrease in equilibrium constants. For example, the equilibrium constants for the complexes decrease by factors of 140, 550, and 40 as the ligands are changed from I to V, II to VI, and III to VII, respectively.

The decreased reactivity for this series of complexes could be due to the enhanced $\sigma$-donor ability of the nitrogen in the pyridine ring via inductive effects caused by the methyl group and/or increased steric effects caused by the methyl groups. When the Mulliken charges on $\mathrm{Ni}$ are calculated for the complexes of V, VI, and VII, it seems that the methyl groups $\alpha$ to the pyridine nitrogen do have an inductive effect, but the reduced reactivity of these complexes is also a consequence of increased sterics. One piece of evidence in support this contention is the similarity in Mulliken charges between the Ni complexes of Ligand III and Ligands V and VI, but the noticeably different equilibrium constants. Another piece of evidence comes from a comparison of the $\mathrm{Ni}(\mathrm{II})$ complexes of Ligands IV and VII, which have similar Mulliken charges on the metal, but equilibrium constants that differ by about a factor of five.

The complexes of Ligands V, VI, and VII seem then to have lower than expected equilibrium constants based upon the relationship between the Mulliken charge and the equilibrium constants seen for the complexes of Ligands I, III, and IV. The lower than expected equilibrium constants are probably due to the steric bulk of the methylpyridine groups, which prevent $\mathrm{CH}_{3} \mathrm{CN}$ from optimally binding to the metal. A structural comparison of the complexes of Ligands I and V (on the left and right, respectively, in Figure 3) illustrates the steric hindrance caused by the methylpyridine groups. In the $\mathrm{CH}_{3} \mathrm{CN}$ adduct of the Ni complex of Ligand I (structure not shown), $\mathrm{CH}_{3} \mathrm{CN}$ attaches to the complex via the open space that is available trans to one of the aliphatic nitrogens, which is circled in Figure 3. The same space in the $\mathrm{Ni}$ complex of Ligand $\mathrm{V}$ is obstructed by the methyl group attached to the pyridine ring, which hinders optimal orientation of $\mathrm{CH}_{3} \mathrm{CN}$ with respect to the metal and thus weakens the metal$\mathrm{CH}_{3} \mathrm{CN}$ interaction.

Placing methyl groups on the amine donor atoms might also be expected to have an effect on reactivity because of inductive effects, but it is unclear if steric effects would also affect the reactions of such complexes. Tertiary amines are usually weaker ligands than secondary amines in solution due to solvation effects, but gas-phase results typically indicate increased metalligand binding strengths as the degree of alkylation is increased, presumably because of greater ligand $\sigma$-donation [54,55]. The collection of ligands used in this study does not allow for a thorough evaluation of the effect of amine methylation, but some insight is gath-
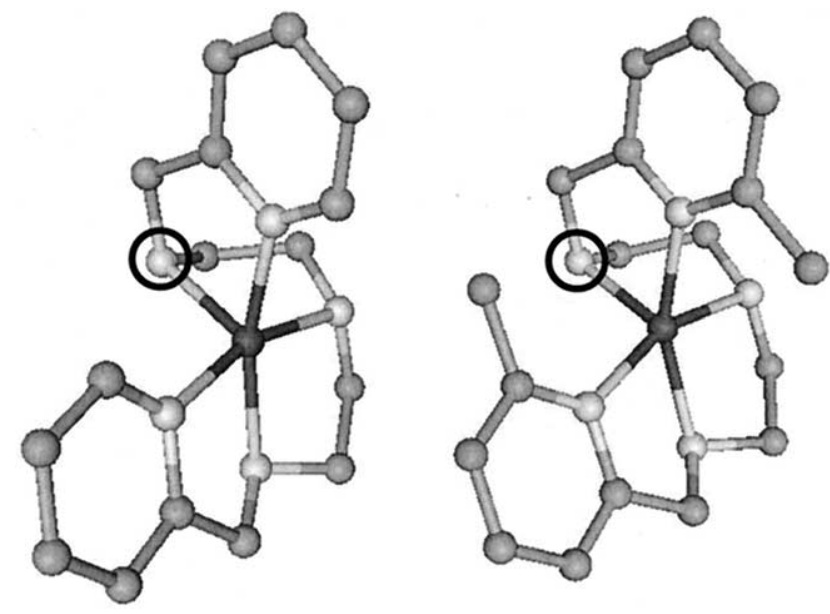

Figure 3. Minimum energy structures, which were obtained by DFT calculations (B3LYP/LANL2DZ), for the Ni complexes of the Ligands I (left) and $\mathrm{V}$ (right). In the $\mathrm{CH}_{3} \mathrm{CN}$ adducts of these complexes, $\mathrm{CH}_{3} \mathrm{CN}$ binds to the metal trans to the circled $\mathrm{N}$ atoms.

ered by comparing the metal complex reactivity of the following ligands: VIII versus VII, IX versus III, and X versus I.

A comparison of the complexes of Ligands VIII and VII shows that methylation of one of the amine groups (i.e., Ligand VIII) does not change the reactivity of the complex much. The lower reactivity of the complexes of these ligands when compared with the complexes of Ligand III, though, suggests that methylation of the pyridine ring has more of an impact on reactivity than amine methylation. In a similar manner the equilibrium constants for the complexes of IX are only slightly lower than the complexes of Ligand III. Indeed, the Mulliken charge on $\mathrm{Ni}(\mathrm{II})$ in the complex of Ligand IX is only slightly smaller than the value for the complex of Ligand III, and the resulting decrease in complex reactivity falls right in line with the trend observed for complexes minimally affected by sterics (i.e., complexes of Ligands I-IV).

Methylating all of the amine groups in the ligand (i.e., Ligand X) results in lower equilibrium constants as is evident from a comparison of the complexes of Ligand $X$ and Ligand I. In this case, though, inductive effects cannot fully explain the drastic drop in the equilibrium constants in going from Ligand I to Ligand $X$. The Mulliken charge on the Ni(II) complex of Ligand $\mathrm{X}$ is lower than on the $\mathrm{Ni}(\mathrm{II})$ complex of Ligand I, but the decrease is not enough to explain the fact that complexes of Ligand $\mathrm{X}$ exhibit the lowest equilibrium constant of all the complexes studied. Very likely steric effects also play an important role in reducing the reactivity of this complex.

\section{Conclusions}

In summary, ion-molecule reactions of $\mathrm{CH}_{3} \mathrm{CN}$ with a series $\mathrm{Ni}$ (II) complexes have been performed. The ligands in these complexes differ by the placement of one 
or more methylene and/or methyl groups so that chelate ring size, sterics, and inductive effects can be examined in the gas phase. A decrease in equilibrium constants is observed as the chelate ring size increases from five to seven. The decreased gas-phase reactivity arises from greater ligand flexibility, which leads to better overlap of the ligand donor atoms with the metal. This conclusion is supported by DFT calculations, which show decreased Mulliken charges on the metal as the chelate ring size increases. These results, however, somewhat contrast with previous predictions based on solution data, which speculate that increased ligand strain and poorer metal-donor overlap are present as the chelate ring size increases. Further experiments of the type described in this work may provide more insight into how chelate ring size affects the stability constants of metal complexes in solution. Additional gas-phase investigations of complexes that have been well studied in solution with respect to chelate ring size would first be necessary to confirm the generality of the trend observed here. Assuming that the generality observed here holds, gas-phase measurements involving complexes with ligands that have different solvation properties and complexes with larger metal ions might help clarify the effect of chelate ring size.

In general, rate and equilibrium constants also decrease for the metal complex reactions with $\mathrm{CH}_{3} \mathrm{CN}$, as methyl groups are added on or next to nitrogen donor atoms. Both inductive and steric effects are responsible for these results. Because one eventual goal of these ion-molecule reaction studies is to use such reactions as indicators of divalent metal complex coordination structure, the effect of sterics on these reactions is important. Previous studies [12, 18-20,36] have shown that metal electronic structure and a complex's coordination sphere have a profound effect on reactivity. The present study, however, indicates that ion-molecule reactions are also strongly influenced by steric factors, perhaps even to an extent that previously observed correlations between coordination structure and gasphase reactivity may be obscured in cases where the metal is sterically hindered.

\section{Acknowledgments}

MYC thanks the Schering-Plough Research Institute for a summer research fellowship.

\section{References}

1. Alvarez, E. J.; Vartanian, V. H.; Brodbelt, J. S. Anal. Chem. 1997, 69, 1147-1155.

2. Hopfgartner, G.; Piguet, C.; Henion, J. D. J. Am. Soc. Mass Spectrom. 1994, 5, 748-756.

3. Gatlin, C. L.; Turecek, F.; Vaisar, T. J. Am. Chem. Soc. 1995, 117, 3637-3638.

4. Gatlin, C. L.; Turecek, F.; Vaisar, T. J. Mass Spectrom. 1995, 30, 1605-1616.

5. Gatlin, C. L.; Turecek, F.; Vaisar, T. J. Mass Spectrom. 1995, 30, $1617-1627$.
6. Kohler, M.; Leary, J. A. J. Am. Soc. Mass Spectrom. 1997, 8, $1124-1133$.

7. Sible, E. M.; Brimmer, S. P.; Leary, J. A. J. Am. Soc. Mass Spectrom. 1997, 8, 32-42.

8. Domingues, M. R. M.; Nemirovskiy, O. V.; Marques, M. G. O. S.; Neves, M. G.; Cavaleiro, J. A. S.; Ferrer-Correia, A. J.; Gross, M. L. J. Am. Soc. Mass Spectrom. 1998, 9, 767-774.

9. Chu, I. K.; Lau, T. C.; Siu, K. M. W. J. Mass Spectrom. 1998, 33, $811-818$.

10. Shen, J.; Brodbelt, J. S. Analyst 2000, 125, 641-650.

11. Desaire, H.; Beyer, M. K.; Leary, J. A. J. Am. Soc. Mass Spectrom. 2001, 12, 528-536.

12. Vachet, R. W.; Hartman, J. R.; Callahan, J. H. J. Mass Spectrom. 1998, 12, 1209-1225.

13. Hartman, J. R.; Vachet, R. W.; Callahan, J. H. Inorg. Chim. Acta. 2000, 297, 79-87.

14. Vachet, R. W.; Callahan, J. H. J. Mass Spectrom. 2000, 35, 311-320.

15. Vachet, R. W.; Hartman, J. R.; Gertner, J. W.; Callahan, J. H. Int. J. Mass Spectrom. 2001, 204, 101-112.

16. Hartman, J. R.; Vachet, R. W.; Pearson, W.; Wheat, R. J.; Callahan, J. H. Inorg. Chim. Acta 2003, 343, 119-132.

17. Hartman, J. R.; Combariza, M. Y.; Vachet, R. W. Inorg. Chim. Acta 2004, 357, 51-58.

18. Combariza, M. Y.; Vachet, R. W. J. Am Soc. Mass Spectrom. 2002, 13, 813-825.

19. Combariza, M. Y., Fermann, J. T., Vachet, R. W. Inorg. Chem. 2004, 43, 2745-2753.

20. Combariza, M. Y.; Vachet, R. W. J. Phys. Chem. A 2004, 108, 1757-1763.

21. Chaparro, A. L.; Vachet, R. W. J Mass. Spectrom. 2003, 38, 333-342.

22. Shen, N. Z.; Pope, R. M.; Dearden, D. V. Int. J. Mass Spectrom. 2000, 196, 639-652.

23. Frisch, M. J.; Trucks, G. W.; Schlegel, H. B.; Scuseria, G. E.; Robb, M. A.; Cheeseman, J. R.; Zakrzewski, V. G.; Montgomery, J. A., Jr.; Stratmann, R. E.; Burant, J. C.; Dapprich, S. J.; Millam, M.; Daniels, A. D.; Kudin, K. N.; Strain, M. C.; Farkas, O.; Tomasi, J.; Barone, V.; Cossi, M.; Cammi, R.; Mennucci, B.; Pomelli, C.; Adamo, C.; Clifford, S.; Ochterski, J.; Petersson, G. A.; Ayala, P. Y.; Cui, Q.; Morokuma, K.; Malick, D. K.; Rabuck, A. D.; Raghavachari, K.; Foresman, J. B.; Cioslowski, J.; Ortiz, J. V.; Stefanov, B. B.; Liu, G.; Liashenko, A.; Piskorz, P.; Komaromi, I.; Gomperts, R.; Martin, R. L.; Fox, D. J.; Keith, T.; Al-Laham, M. A.; Peng, C. Y.; Nanayakkara, A.; Gonzalez, C.; Challacombe, M. P.; Gill, M. W.; Johnson, B.; Chen, W.; Wong, M. W.; Andres, J. L.; Head-Gordon, M.; Replogle, E. S.; Pople, J. A. Gaussian 98, Revision A.3; Gaussian, Inc: Pittsburgh PA, 1998.

24. Pople, J. A.; Head-Gordon, M.; Fox, D.J.; Raghavachari, K.; Curtiss, L. A. J. Chem. Phys. 1989, 90, 5622-5629.

25. Curtiss, L. A.; Jones, C.; Trucks, G. W.; Raghavachari, K.; Pople, J. A. J. Chem. Phys. 1990, 93, 2537-2545.

26. Becke, A. D. J. Chem. Phys. 1993, 98, 5648-5652.

27. Dunning, T. H., Jr.; Hay, P. J. Schaefer, H.F. III., Ed.; In Modern Theoretical Chemistry, Vol. III; Plenum Press: New York, NY, 1976; pp 1-28.

28. Hay, P. J.; Wadt, R. J. Chem. Phys. 1985, 82, 270-283.

29. Hay, P. J.; Wadt, R. J. Chem. Phys. 1985, 82, 284-298.

30. Hay, P. J.; Wadt, R. J. Chem. Phys. 1985, 82, 299-310.

31. Mulliken, R. S. J. Chem. Phys. 1955, 23, 2338-2342.

32. Mulliken, R. S. J. Chem. Phys. 1955, 23, 2343-2346.

33. Mulliken, R. S. J. Chem. Phys. 1955, 23, 1833-1840.

34. Mulliken, R. S. J. Chem. Phys. 1955, 23, 1841-1846.

35. Hall, G. G. Adv. At. Mol. Phys. 1985, 20, 41-63.

36. Combariza, M. Y.; Vachet, R. W. Anal. Chim. Acta 2003, 496, 233-248. 
37. Hancock, R. D.; Martell, A. E. Supramol. Chem. 1996, 6, 401-407. 38. Hay, B. P.; Hancock, R. D. Coord. Chem. Rev. 2001, 212, 61-78.

39. Hancock, R. D.; Martell, A. E. Chem. Rev. 1989, 89, 1875-1914.

40. Martell, A. E.; Smith, R.M. Critical Stability Constants, Vols. I-VI; Plenum Press: New York, NY, 1974, 1974, 1975, 1976, 1977, 1982, 1989.

41. Hancock, R. D.; Ngwenya, M. P. J. Chem. Soc. Dalton Trans. 1987, 2911-2915.

42. Anderegg, G; Bläuenstein, P. Helv. Chim. Acta 1982, 65, 913-923.

43. Hancock, R. D.; Wade, P. W.; Ngwenya, M. P.; de Sousa, A. S.; Damu, K. V. Inorg. Chem. 1990, 29, 1968-1974.

44. Cukrowski, I.; Cukrowska, E.; Hancock, R. D.; Anderegg, G. Anal. Chim. Acta 1995, 312, 307-321.

45. Chanston, T. E.; Hancock, R. D. Inorg. Chim. Acta 1995, 230, 165-167.
46. Hancock, R. D. Pure Appl. Chem. 1986, 58, 1445-1452.

47. Fahey, A. M.; Vachet, R. W., unpublished.

48. Pereira, B. A.; Ince, M. P.; Talaty, E. R.; Van Stipdonk, M. J. Rapid Commun. Mass Spectrom. 2001, 15, 615-622.

49. Pereira, B. A; Gallardo, A. L.; Barr, J. M.; Tekarli, S. M.; Anbalagan, V.; Talaty, E. R.; Van Stipdonk, M. J. J. Mass Spectrom. 2002, 37, 401-413.

50. Shen, J.; Brodbelt, J. Int. J. Mass Spectrom. 1998, 176, 39-61.

51. Shen, J.; Brodbelt, J. J. Am. Soc. Mass Spectrom. 1999, 10, $126-135$.

52. Shen, J.; Brodbelt, J. J. Mass Spectrom. 1999, 34, 137-146.

53. Schwarzenbach, G. Helv. Chim. Acta 1952, 35, 2344-2363.

54. Deng, H. T.; Kebarle, P. J. Phys Chem. A 1998, 102, 571-579.

55. Kappes, M. M.; Staley, R. H. J. Am. Chem. Soc. 1982, 104, 1813-1819. 